Rapid Reviews COVID-19

\title{
Review 2: "The effect of eviction moratoriums on the transmission of SARS- CoV-2"
}

Srini Venkatramanan ${ }^{1}$

${ }^{1}$ University of Virginia, Biocomplexity Institute, USA

Published on: Feb 22, 2021

License: Creative Commons Attribution 4.0 International License (CC-BY 4.0). 


\section{$\underline{\text { RR:C19 Evidence Scale rating by reviewer: }}$}

- Reliable. The main study claims are generally justified by its methods and data. The results and conclusions are likely to be similar to the hypothetical ideal study. There are some minor caveats or limitations, but they would/do not change the major claims of the study. The study provides sufficient strength of evidence on its own that its main claims should be considered actionable, with some room for future revision.

\section{Review:}

The authors use computational models to simulate counterfactual scenarios under different assumptions about eviction moratoria (continued enforcement vs. resuming at different rates). Their models show that across scenarios, evictions lead to more infections, and they quantify the exact extent using realistic parameters and population assumptions with Philadelphia as an example. The proposed approach is useful for understanding the impact of such policy decisions at a fine geographic scale and will help policymakers incorporate the epidemiological impacts when deciding on lifting eviction moratoria.

Especially interesting are the different measures used by the authors to quantify the impact, which may be important in influencing public perceptions:

- increase in individual risk of infection (those evicted/doubled-up, and in general)

- excess infections/eviction

The authors capture the impact of evictions by perturbing the household distribution (shifting it upward) to capture the phenomenon of 'doubling-up'. I think this is a reasonable assumption with the following caveats (may not change the qualitative results):

- Validating the extent of this reconfiguration will be difficult for a given city. It will depend on existing social networks and the demographic (racial/ethnic/age/income) profiles. The authors make a reasonable assumption about it for heterogeneous eviction rates, social distancing measures.

- While spillovers such as an increase in homeless shelters/encampments are included in supplemental analyses, migration out of the city is not considered.

- For the evicted household (mostly triggered due to unemployment), their external contacts (e.g., 
The authors also use Cuebiq data to infer home location change from February 2020 to October 2020. More details on this could help characterize the actual impact of evictions on household reconfiguration. Figure S16 shows individuals 10-30\% moving users of a particular cluster go to another.

The additional supplemental analyses cover a wide range of additional assumptions and characterize the sensitivity to assumed parameters. The discussion is also quite detailed and positions the qualitative results in the context of other literature. 